\title{
Sitosterolemia: a review and update of pathophysiology, clinical spectrum, diagnosis, and management
}

\author{
Eun-Gyong Yoo, MD, PhD \\ Department of Pediatrics, \\ CHA Bundang Medical Center, \\ CHA University, Seongnam, Korea
}

\begin{abstract}
Sitosterolemia is an autosomal recessive disorder characterized by increased plant sterol levels, xanthomas, and accelerated atherosclerosis. Although it was originally reported in patients with normolipemic xanthomas, severe hypercholesterolemia have been reported in patients with sitosterolemia, especially in children. Sitosterolemia is caused by increased intestinal absorption and decreased biliary excretion of sterols resulting from biallelic mutations in either ABCG5 or $A B C G 8$, which encode the sterol efflux transporter ABCG5 and ABCG8. Patients with sitosterolemia show extreme phenotypic heterogeneity, ranging from almost asymptomatic individuals to those with severe hypercholesterolemia leading to accelerated atherosclerosis and premature cardiac death. Hematologic manifestations include hemolytic anemia with stomatocytosis, macrothrombocytopenia, splenomegaly, and abnormal bleeding. The mainstay of therapy includes dietary restriction of both cholesterol and plant sterols and the sterol absorption inhibitor, ezetimibe. Foods rich in plant sterols include vegetable oils, wheat germs, nuts, seeds, avocado, shortening, margarine and chocolate. Hypercholesterolemia in patients with sitosterolemia is dramatically responsive to low cholesterol diet and bile acid sequestrants. Plant sterol assay should be performed in patients with normocholesterolemic xanthomas, hypercholesterolemia with unexpectedly good response to dietary modifications or to cholesterol absorption inhibitors, or hypercholesterolemia with poor response to statins, or those with unexplained hemolytic anemia and macrothrombocytopenia. Because prognosis can be improved by proper management, it is important to find these patients out and diagnose correctly. This review article aimed to summarize recent publications on sitosterolemia, and to suggest clinical indications for plant sterol assay.
\end{abstract}

Keywords: Sitosterolemia, Phytosterolemia, Hypercholesterolemia, Plant sterol
Received: 14 March, 2016 Accepted: 15 March, 2016

Address for correspondence: Eun-Gyong Yoo, MD, PhD

Department of Pediatrics, CHA Bundang Medical Center, $\mathrm{CHA}$ University, 59 Yatap-ro, Bundang-gu, Seongnam 13496, Korea

Tel: +82-31-780-1999

Fax: +82-31-780-5239

E-mail: pedyoo@cha.ac.kr

\section{Introduction}

Sitosterolemia, also known as phytosterolemia, is an autosomal recessive disorder characterized by increased plant sterol levels, xanthomas, and accelerated atherosclerosis ${ }^{1,2)}$. It is caused by increased intestinal absorption and decreased biliary excretion of plant sterols resulting from homozygous or compound heterozygous mutations in either ABCG5 or ABCG8, which encode the sterol efflux transporter ABCG5 (sterolin-1) and ABCG8 (sterolin-2) that pumps sterols out to intestinal lumen or into bile $\mathrm{e}^{3,4}$. Although it is a rare disease, it is an important disease that led to understanding of the physiologic pathway about sterol influx and efflux ${ }^{5,6)}$.

Mediterranean stomatocytosis/macrothrombocytopenia has been identified as the hematological presentation of sitosterolemia ${ }^{7)}$. Stomatocytic hemolysis, large platelets, splenomegaly, and abnormal bleeding can be associated, and hematologic manifestations can be the only clinical sign of sitosterolemia ${ }^{8)}$. The true prevalence of sitosterolemia is unknown due to 
underdiagnosis, and sitosterolemia may be more frequent than previously thought. One Asian individual with sitosterolemia was identified incidentally out of 2,542 persons from a study in which plasma plant sterols were analyzed ${ }^{9)}$.

Because delayed diagnosis can lead to poor clinical outcome due to advanced atherosclerotic cardiovascular disease and prognosis can be improved by proper management including plant sterol restriction and cholesterol absorption inhibitor in sitosterolemia, it is important to find these patients out and diagnose correctly ${ }^{2,8,10}$. This review article aimed to summarize recent publications on the pathophysiology, clinical spectrum, diagnosis and management of sitosterolemia, and to suggest clinical indications for plant sterol assay.

\section{The plant sterols}

Plant sterols are rich in vegetable oils, wheat germs, nuts, seeds, avocados, chocolate, and margarine ${ }^{2,6}$. They are structurally very similar to cholesterol, but they differ by the presence of an ethyl or methyl group (sitosterol and campesterol, respectively) or a double bond (stigmasterol $)^{1}$. Sitosterol is usually the most abundant plant sterol in the diet and the predominant form found in patients with sitosterolemia ${ }^{8,11)}$.

Average Western diet contains similar amount of cholesterol and plant sterols. Although approximately $50 \%$ of dietary cholesterol is absorbed, less than $5 \%$ of plant sterols are absorbed in normal individuals ${ }^{6,11)}$. High plant sterol diet was extremely toxic in animal models of sitosterolemia, and it was suggested that the mammalian body defends itself against plant sterols because they are toxic when accumulated, although similar toxicity have not been documented in human yet ${ }^{12,13)}$.

It is clear that plant sterols are toxic to those with sitosterolemia, but plant sterol intake seems to be safe to nonsitosterolemic individuals. Instead, plant sterols can competitively inhibit cholesterol absorption, and the cholesterol lowering effect of plant sterols have been documented ${ }^{11}$. Although they have not been shown to reduce clinical outcomes, many cholesterol lowering functional foods are enriched with plant sterols ${ }^{14,15)}$. On the other hand, studies have raised the possibility of association between plant sterol levels and atherosclerosis ${ }^{16,17}$. The cholesterol lowering effect may compensate the potential risk of increased plant sterol intake ${ }^{9,11)}$, and the debate whether plant sterol is beneficial or harmful is still ongoing ${ }^{15,18)}$.

\section{Sterol absorption in normal subjects}

Dietary cholesterol and noncholesterol sterols, mainly plant sterols and stanols (saturated sterols), are absorbed form the intestinal lumen via the sterol influx transporter, Nieman Pick C1 Like 1 (NPC1L1) $)^{6}$. The NPC1L1, the gatekeeper of sterol absorption, have lower affinity to plant sterols than cholesterol ${ }^{19,20)}$. After absorption to the enterocytes, about $50 \%-60 \%$ of cholesterol is esterified by the acetyl-sterol O-acyltransferase 2 (SOAT2) and transported to liver packed in the chylomicrons ${ }^{21}$. Unesterified cholesterol or plant sterols are pumped back to intestinal lumen by ABCG5/ABCG8, the sterol efflux transporters ${ }^{6}$. The SOAT2 also have low affinity with plant sterols, allowing preferential plant sterol efflux by the ABCG5/ABCG8 ${ }^{22)}$. Plant sterols not pumped back to the intestinal lumen become part of the chylomicrons, transported to the liver, and eventually pumped out into the bile by the hepatic ABCG5/ABCG8 transporters ${ }^{6,11)}$.

\section{Disrupted sterol homeostasis in sitosterolemia}

Biallelic defects in either $A B C G 5$ or $A B C G 8$ result in increased intestinal absorption and decreased biliary excretion of plant sterols, leading to extremely high plasma levels of plant sterols ${ }^{3,4)}$. Patients with sitosterolemia absorb $15 \%$ to $60 \%$ of ingested sitosterol, which lead to a 50- to 200-fold increase in their plasma sitosterol levels ${ }^{1,3)}$. Plant sterols comprise $15 \%$ to $20 \%$ of total plasma sterols in patients with sitosterolemia and are carried in low-density lipoprotein (LDL) and very-LDL particles ${ }^{3,23)}$.

In a patient with liver failure and sitosterolemia that underwent liver transplantation, the elevated plant sterol levels decreased to values less than $1 / 10$ of pretransplantation level, suggesting that the liver functions as the predominant organ for maintaining sterol balance ${ }^{24)}$. ABCG5/ABCG8 expression either in liver or intestine protected animals from sterol accumulation in a recent study ${ }^{25}$.

Although sterol absorption was moderately increased in heterozygotes, they are asymptomatic with normal cholesterol levels and normal to slightly increased plant sterol levels ${ }^{1,26)}$.

\section{Clinical spectrum of sitosterolemia}

Patients with sitosterolemia show extreme phenotypic heterogeneity. Whereas some patients with homozygous mutations are almost totally asymptomatic, others show severe hypercholesterolemia leading to accelerated atherosclerosis and premature cardiac death ${ }^{1,27-30)}$. A 10-year-old girl from Iran, who had received almost vegetable-free diet in Iran and started to intake much more vegetables and olive oil after her family moved to Europe, developed xanthomas and hypercholesterolemia in a short period of time and was finally diagnosed for sitosterolemia ${ }^{31}$. Although the amount of dietary plant sterol intake should be at least partially related with the severity of clinical disease, the mechanism of phenotypic heterogeneity, even between the family members that shares same gene and environment, is not fully understood yet. A recent report in a Chinese family with sitosterolemia suggested potential effects of NPC1L1 polymorphisms in protecting against clinical disease ${ }^{29)}$. Major clinical features of sitosterolemia, especially in young patients with sitosterolemia are summarized in Table 1. 
Table 1. Clinical spectrum of sitosterolemia

\begin{tabular}{lc}
\hline Clinical spectrum of sitosterolemia & References \\
\hline Asymptomatic (with or without hypercholesterolemia) & $28-30$ \\
Xanthomas (with or without hypercholesterolemia) & \\
Tendinous or tuberous xanthomas on extensor sites & $1,10,23,27,28$ \\
Planar xanthomas in the creases of Achilles region & 33 \\
Intertriginous xanthomas & 35 \\
Hypercholesterolemia & \\
Mild to moderate hypercholesterolemia & $10,28,31,44$ \\
Severe hypercholesterolemia ( $\geq 500$ mg/dL) & $32,33,35,47$ \\
Premature cardiovascular disease & \\
Premature coronary heart disease (at age 16-29) & 10,29 \\
Sudden cardiac death (at age 5-18) & $22,47,48$ \\
Hematologic manifestations & \\
Hemolytic anemia with stomatocytosis & $7,8,52,54$ \\
Macrothrombocytopenia & $7,8,52,54$ \\
Splenomegaly & 8,53 \\
Abnormal bleeding & 55 \\
Arthritis, arthralgia & 2 \\
Hepatic failure* & 24 \\
\hline
\end{tabular}

*Not confirmed whether hepatic failure was due to sitosterolemia or not.

\section{Hypercholesterolemia}

Although it was originally reported in patients with normolipemic xanthomas ${ }^{27)}$, cholesterol absorption is also increased in patients with sitosterolemia, and serum cholesterol levels are usually elevated ${ }^{1,10)}$. Very high levels of cholesterol (up to 1,000 $\mathrm{mg} / \mathrm{dL}$ ) have been reported in patients with sitosterolemia, especially in children ${ }^{32,33)}$. Immature intestine may absorb higher amounts of cholesterol compared with that of adults ${ }^{34)}$.

Breastfed infants with sitosterolemia show unique clinical features ${ }^{32,33,35)}$. The plant sterol intake of a breastfed infant should be minimal because the plasma sitosterol levels of the heterozygote mother should be only slightly increased. However their cholesterol intake can be high due to high cholesterol content of human milk (90-150 mg/L vs. 0-4 mg/L in human milk and infant formula, respectively $)^{36)}$. Breastfed infants with sitosterolemia can present with extremely high cholesterol levels with xanthomatosis, but with normal sitosterol:cholesterol ratio due to only mildly elevated plant sterol levels ${ }^{33)}$. The plant sterol level increase and the cholesterol level somewhat decrease as the infant start taking fruits and vegetables ${ }^{33,35)}$.

\section{Xanthomas}

Tendinous or tuberous xanthomas on extensor areas, such as Achilles tendon, extensor tendons of the hand, elbows and knees are the major clinical manifestations of sitosterolemia ${ }^{1,23,27)}$. Minor trauma plays an important role in the development of xanthomas, and this is why they appear on extensor surfaces in most patients ${ }^{37)}$.
Xanthomatosis is rarely observed in young children, and when present, homozygous familial hypercholesterolemia (FH) or autosomal recessive hypercholesterolemia is most often suspected $^{37,38)}$. Xanthomas may begin to appear at very young age in sitosterolemia, sometimes during the first year of life ${ }^{33,35)}$.

Intertriginous xanthomas are a very rare type of planar xanthomas and have been reported to be pathognomonic for homozygous $\mathrm{FH}^{39)}$. However, intertriginous xanthomas (first noticed at the age of 3 months when the patient was being exclusively breastfed) were observed in a 15-month-old Korean girl with sitosterolemia, suggesting that intertriginous xanthomas may develop in young children with extremely high cholesterol levels of any etiology ${ }^{35)}$. Friction between the skins in intertriginous areas may contribute to the development of xanthomas in a chubby infant in whom extensor areas are relatively spared because movement is not active yet.

Most sitosterolemic patients with severe atherosclerotic cardiovascular disease also showed xanthomas ${ }^{1,10,23,28)}$. Xanthomas evolve as clusters of foam cells in the skin, and the mechanisms involved in the development of xanthoma seem to be similar to those in early stages of atherosclerotic plaques ${ }^{40)}$. According to a meta-analysis on patients with genetic diagnosis of FH, the presence of tendon xanthomas was associated with a 3.2 times higher risk of cardiovascular disease ${ }^{41)}$. Xanthelasma of the eyelids was considered to be only a cosmetic lesion until recently, however recent prospective studies showed that it is connected with an increased cardiovascular risk and reduced average lifespan ${ }^{42)}$. In contrast to the initial case with normolipemic xanthomas ${ }^{27)}$, xanthomas regress and sometimes completely disappear in some patients with sitosterolemia, usually associated with dramatically decreased plasma cholesterol levels, although plant sterol levels were still relatively high $^{32,35,43-46)}$.

\section{Atherosclerotic cardiovascular disease}

Some patients with sitosterolemia develop premature atherosclerosis leading to sudden cardiac death at as early as $5^{47)}, 13^{48)}, 18^{22)}$ years of age, whereas others, even in the same family of symptomatic patients, do not show any classic sign of sitosterolemia $^{24,28,35)}$.

Both elevated plasma cholesterol and plant sterol levels can contribute to the premature vascular disease in patients with sitosterolemia. Accumulation of plant sterol in plasma lipoproteins influences the stability of both cholesterol and plant sterol in lipoproteins, favoring the accumulation of these sterols within tissues, initiating inflammatory reactions, and may cause premature atherosclerosis ${ }^{6}$.

Coronary plaque disruption and superimposed thrombosis is the major cause of acute myocardial infarction and sudden cardiac death ${ }^{49)}$. The composition and vulnerability of plaque rather than its volume or the severity of stenosis are more important for the development of the thrombus-mediated acute coronary syndromes ${ }^{49)}$. Plant sterols are relatively poorly 
esterified by the sterol-esterifying enzyme acyl-CoA-cholesterol acyl transferase. Macrophages incubated with sitosterolcontaining lipoproteins accumulated free sterols and underwent necrotic cell death, which may contribute to the formation of rupture-prone plaque ${ }^{50)}$.

Premature coronary heart disease can develop in sitosterolemic patients with normal cholesterol levels. A 16-year-old sitosterolemic girl with normal cholesterol level was reported to have premature coronary heart disease requiring coronary bypass grafts $^{51)}$, and a normocholesterolemic patient who underwent a 3 vessel coronary bypass surgery at the age of 29 was diagnosed with sitosterolemia after that ${ }^{10)}$.

On the other hand, Hansel et al. ${ }^{30)}$ could not find significant signs of premature atherosclerosis in 5 patients with sitosterolemia aged 11 to 21 years, in spite of severe hypercholesterolemia as well as extremely high plant sterol levels. They suggested that the premature atherosclerosis in some patients with sitosterolemia may be due at least in part to mechanisms independent of elevated circulating plant sterol levels ${ }^{30)}$.

\section{Hematologic manifestations}

Rees et al. ${ }^{7}$ revealed that stomatocytic hemolysis and macrothrombocytopenia (previously known as the Mediterranean stomatocytosis or Mediterranean macrothrombocytopenia, which had been a poorly understood hematological condition) is the hematological presentation of sitosterolemia.

Stomatocysis, hemolytic anemia, thrombocytopenia with very large platelets, splenomegaly, and abnormal bleeding can be associated with sitosterolemia ${ }^{8)}$. Because the $A B C G 5$ and $A B C G 8$ are only expressed in intestine and liver, acquired accumulation of circulating plant sterols and their incorporation into red blood cells (RBC) and platelet seems to be resulting in abnormal morphology and function ${ }^{7}$.

Blood cells can be a main target for the toxic effect of plasma plant sterols, and sitosterolemia can be manifested mainly by hematologic abnormalities ${ }^{52}$. Three patients from a Chinese family, all of whom had suffered from severe hemolytic anemia and macrothrombocytopenia since 3 to 4 years of age and underwent splenectomy in their 10's, was diagnosed as sitosterolemia in their 20's. All of these patients had increased plasma sitosterol but normal cholesterol levels ${ }^{52}$. Thirteen sitosterolemic patients with hematologic manifestations, including 2 patients without any classical features of sitosterolemia, had been misdiagnosed with immune thrombocytopenia (ITP), Evans syndrome, or secondary ITP with delay being 15 to 49 years between symptom onset and correct diagnosis ${ }^{53)}$. Plasma plant sterols should be analyzed in patients with unexplained hemolytic anemia with macrothrombocytopenia to avoid unnecessary splenectomy ${ }^{54}$.

Recently, Kanaji et al. ${ }^{55)}$ have identified that the bleeding abnormalities and macrothrombocytopenia associated with sitosterolemia are due to direct plant sterol incorporation into the platelet membrane, resulting in platelet hyperactivation, reduced $\alpha \operatorname{IIb} \beta 3$ surface expression, loss of the GPIbaFlnA linkage, microparticle formation, and ultimately poor hemostatic functions.

\section{Diagnosis of sitosterolemia}

Routine laboratory methods do not distinguish plant sterols from cholesterol, and a more accurate method such as gas chromatography-mass spectrometry (GC-MS) is required. Measurement of serum plant sterol by GC-MS or liquid chromatography-mass spectrometry is regarded as a reliable test for screening sitosterolemia, in which unequivocally increased plant sterol levels and sitosterol: cholesterol levels are almost invariably observed ${ }^{1)}$.

Genetic confirmation can be given by direct sequencing of exons and intron-exon boundaries of the ABCG5 and ABCG8 genes, each comprised of 13 exons and located in a head-tohead organization on chromosome 2 p21, and documenting the homozygous or compound heterozygous mutations in either $A B C G 5$ or $A B C G 8^{3,4)}$. Asian patients usually have mutations in $A B C G 5$, while Caucasian patients usually have $A B C G 8$ mutations ${ }^{3,32)}$. However, mutations in $A B C G 8$ have been reported in 3 of 8 families with hematologic manifestations of sitosterolemia according to a recent Chinese study, suggesting that $A B C G 8$ mutations are not exclusive to Caucasians ${ }^{53}$. DNA sequencing of $A B C G 5 / A B C G 8$ is should be performed to rule out sitosterolemia in breastfed infants, because they can exhibit only mild elevation of plasma sitosterol level and normal sitosterol:cholesterol ratio ${ }^{33}$.

In contrast to patients with homozygous FH that are relatively refractory to dietary modification and cholesterol-lowering agents, plasma cholesterol levels in sitosterolemic patients are extremely sensitive to dietary cholesterol restriction and bile acid sequestrants $s^{32,43,44)}$.

The entire pathway of cholesterol biosynthesis including hepatic hydroxymethylglutaryl coenzyme A (HMG CoA) reductase is exceptionally down-regulated in patients with sitosterolemia ${ }^{10,56)}$. It was also reported that stigmasterol and campesterol inhibit activation of sterol regulatory binding protein-2 (SREBP-2), a transcription factor involved in cholesterol biosynthesis, in cultured adrenocortical cells ${ }^{57}$, and that stigmasterol, not sitosterol, inhibits processing of SREBP-2 leading to reduced cholesterol synthesis in mice ${ }^{58}$.

In nonsitosterolemic individuals, cholesterol synthesis increases after sterol depletion, limiting the effect of sterol absorption inhibitor or bile acid sequestrant ${ }^{57}$. However, there is no such compensatory increase in cholesterol synthesis in those with sitosterolemia, resulting in dramatic reduction in plasma cholesterol levels ${ }^{59)}$. Sitosterolemia should be suspected when the plasma cholesterol falls more than $40 \%$ on a low-cholesterol diet.

Sitosterolemia seems to be significantly underdiagnosed, and many of these patients should be continuing to intake large amount of plant sterols, not knowing that the plant sterols are 'toxic' to them, but believing that those food are 
good for their health. Sitosterolemia might also be significantly underdiagnosed in children in whom screening for lipid profiles is not universally performed. Recent guidelines recommend screening all children at 9-11 years and again at 17-21 years to find those with hypercholesterolemia ${ }^{60)}$. Some of those screened may in fact have sitosterolemia, and these patients may be distinguished by either remarkable response to dietary modification or poor response to statins ${ }^{35}$.

\section{Management of sitosterolemia}

Management of sitosterolemia aims to reduce plasma plant sterol (as low as possible; although perfect control [sitosterol level $<1 \mathrm{mg} / \mathrm{dL}$ ] cannot be achieved) and cholesterol concentrations and to prevent or reduce xanthomas and atherosclerotic cardiovascular diseases ${ }^{2}$.

Mainstay of therapy is dietary restriction of both cholesterol and plant sterols. Foods rich in plant sterols include vegetable oils, wheat germs, nuts, seeds, avocado, most of which are known to be heart-healthy foods ${ }^{2,61)}$. Margarine, shortening, and chocolate should also be avoided. Polished rice should be taken instead of whole grains. Shellfish and seaweeds contain significant amount of algae-derived plant sterols that are also hyperabsorbed in these patients, and they should also be avoided $^{62)}$. However, plant sterol-free diet is almost impossible to accomplish because plant sterols are found in almost every plant-based foods, and low plant sterol diet have resulted in only about $30 \%$ reduction of plasma plant sterol levels ${ }^{11,44)}$.

Pharmacotherapy include the sterol absorption inhibitor, ezetimibe, or bile acid sequestrants such as cholestyramine. Patients with sitosterolemia usually do not respond to statins because HMG CoA reductase activity is already maximally inhibited $^{60)}$

The bile acid sequestrants inhibit the reabsorption of bile acids in the ileum and disrupt the enterohepatic circulation of bile acids. The bile acid sequestrants was reported to reduce plasma plant sterol levels by up to $45 \%$, although they may result in more dramatic decrease in plasma cholesterol levels (50\%$80 \%$ ) and regression of xanthomas ${ }^{43,44)}$. Sitosterolemia should be considered in patients with hypercholesterolemia and/or xanthomas who show dramatic reduction of cholesterol levels or regression of xanthomas by bile acid sequestrant therapy. However, poor compliance and gastrointestinal side effects limit the use of cholestyramine.

Ezetimibe, an inhibitor of intestinal sterol absorption

\section{Table 2. Indications for plant sterol assay}

\section{Indications for plant sterol assay}

Normocholesterolemic xanthomas

Dramatic decrease of cholesterol levels in response to low cholesterol diet and/or bile acid sequestrants or cholesterol absorption inhibitors

Regression of xanthomas, especially with dramatically improved cholesterol levels

Hypercholesterolemia with poor response to statins

Unexplained hemolytic anemia with macrothrombocytopenia through its binding to NPC1L1, is currently considered the choice of treatment for sitosterolemia ${ }^{63)}$. It has been widely used for decreasing serum LDL-cholesterol levels in patients with hypercholesterolemia. Ezetimibe also reduces the intestinal absorption of plant sterols, thereby also lowering plasma plant sterol levels. Ezetimibe alone or in combination with cholestyramine successfully decreased plasma cholesterol and plant sterol levels (about by 50\%; although still much higher than normal values $)^{63)}$, resulting in regression of xanthomas and improvement of carotid bruits and cardiac murmurs in patients with sitosterolemia ${ }^{45)}$. Long-term treatment with ezetimibe 10 $\mathrm{mg}$ /day was safe, tolerable, and effective in reducing plasma plant sterol concentrations in patients with sitosterolemia ${ }^{61,64)}$. Ezetimibe reduced plasma and RBC plant sterol levels, while increasing platelet count and decreasing mean platelet volume, and thereby may reduce the risk for bleeding in sitosterolemia ${ }^{65)}$.

Although pharmacotherapy is usually not performed for children under age 10, an individual with extremely high levels of cholesterol may begin therapy earlier ${ }^{66)}$ Ezetimibe therapy seems to be also safe and effective in children with sitosterolemia, although an infant did not respond to ezetimibe therapy at 7 months of age possibly due to immature glucuronidation system, who finally showed improvement when ezetimibe was restarted at 2 years of age ${ }^{32)}$. Bile acid sequestrants such as cholestyramine can be added for those with insufficient response to ezetimibe ${ }^{2,63)}$.

Arthritis and arthralgia can also be associated with sitosterolemia, and more strict management of sitosterolemia can be helpful' ${ }^{2)}$.

\section{Conclusions}

Plant sterol assay should be performed in patients with normocholesterolemic xanthomas, hypercholesterolemia with unexpectedly good response to dietary modifications or to cholesterol absorption inhibitors, or hypercholesterolemia with poor response to statins, or those with unexplained hemolytic anemia and macrothrombocytopenia (Table 2).

The dramatic cholesterol reduction and regression of xanthomas by proper treatment including plant sterol restriction and cholesterol absorption inhibitor suggest that sitosterolemia can be a controllable condition, and it is important to find these patients out and diagnose correctly because prognosis can be improved by early diagnosis and proper management.

\section{Conflict of interest}

No potential conflict of interest relevant to this article was reported.

\section{References}

1. Salen G, Shefer S, Nguyen L, Ness GC, Tint GS, Shore V. Sitosterolemia. J Lipid Res 1992;33:945-55. 
2. Merkens LS, Myrie SB, Steiner RD, Mymin D. Sitosterolemia. GeneReviews [Internet]. Seattle (WA): University of Washington; 2016 [updated 2013 Apr 4; cited 2016 Feb 29]. Available from: http://www.ncbi.nlm.nih.gov/books/ NBK131810/.

3. Berge KE, Tian H, Graf GA, Yu L, Grishin NV, Schultz J, et al. Accumulation of dietary cholesterol in sitosterolemia caused by mutations in adjacent ABC transporters. Science 2000;290:1771-5.

4. Lu K, Lee MH, Hazard S, Brooks-Wilson A, Hidaka H, Kojima H, et al. Two genes that map to the STSL locus cause sitosterolemia: genomic structure and spectrum of mutations involving sterolin-1 and sterolin-2, encoded by ABCG5 and ABCG8, respectively. Am J Hum Genet 2001;69:278-90.

5. Patel SB. Recent advances in understanding the STSL locus and ABCG5/ABCG8 biology. Curr Opin Lipidol 2014;25:169-75.

6. Othman RA, Myrie SB, Jones PJ. Non-cholesterol sterols and cholesterol metabolism in sitosterolemia. Atherosclerosis 2013;231:291-9.

7. Rees DC, Iolascon A, Carella M, O'marcaigh AS, Kendra JR, Jowitt SN, et al. Stomatocytic haemolysis and macrothrombocytopenia (Mediterranean stomatocytosis/ macrothrombocytopenia) is the haematological presentation of phytosterolaemia. Br J Haematol 2005;130:297-309.

8. Escola-Gil JC, Quesada H, Julve J, Martin-Campos JM, Cedo L, Blanco-Vaca F. Sitosterolemia: diagnosis, investigation, and management. Curr Atheroscler Rep 2014;16:424.

9. Wilund KR, Yu L, Xu F, Vega GL, Grundy SM, Cohen JC, et al. No association between plasma levels of plant sterols and atherosclerosis in mice and men. Arterioscler Thromb Vasc Biol 2004;24:2326-32.

10. Miettinen TA. Phytosterolaemia, xanthomatosis and premature atherosclerotic arterial disease: a case with high plant sterol absorption, impaired sterol elimination and low cholesterol synthesis. Eur J Clin Invest 1980;10:27-35.

11. Izar MC, Tegani DM, Kasmas SH, Fonseca FA. Phytosterols and phytosterolemia: gene-diet interactions. Genes Nutr 2011;6:17-26.

12. Solca C, Tint GS, Patel SB. Dietary xenosterols lead to infertility and loss of abdominal adipose tissue in sterolindeficient mice. J Lipid Res 2013;54:397-409.

13. McDaniel AL, Alger HM, Sawyer JK, Kelley KL, Kock ND, Brown JM, et al. Phytosterol feeding causes toxicity in ABCG5/G8 knockout mice. Am J Pathol 2013;182:1131-8.

14. Calpe-Berdiel L, Mendez-Gonzalez J, Blanco-Vaca F, Carles Escola-Gil J. Increased plasma levels of plant sterols and atherosclerosis: a controversial issue. Curr Atheroscler Rep 2009;11:391-8.

15. Doggrell SA. Lowering LDL cholesterol with margarine containing plant stanol/sterol esters: is it still relevant in 2011? Complement Ther Med 2011;19:37-46.

16. Assmann G, Cullen P, Erbey J, Ramey DR, Kannenberg
F, Schulte H. Plasma sitosterol elevations are associated with an increased incidence of coronary events in men: results of a nested case-control analysis of the Prospective Cardiovascular Münster (PROCAM) study. Nutr Metab Cardiovasc Dis 2006;16:13-21.

17. Helske S, Miettinen T, Gylling H, Mayranpaa M, Lommi J, Turto H, et al. Accumulation of cholesterol precursors and plant sterols in human stenotic aortic valves. J Lipid Res 2008;49:1511-8.

18. Weingärtner O, Teupser D, Patel SB. The atherogenicity of plant sterols: the evidence from genetics to clinical trials. J AOAC Int 2015;98:742-9.

19. Yu L. The structure and function of Niemann-Pick C1-like 1 protein. Curr Opin Lipidol 2008;19:263-9.

20. Kwon HJ, Palnitkar M, Deisenhofer J. The structure of the NPC1L1 N-terminal domain in a closed conformation. PLoS One 2011;6:e18722.

21. Lee RG, Willingham MC, Davis MA, Skinner KA, Rudel LL. Differential expression of ACAT1 and ACAT2 among cells within liver, intestine, kidney, and adrenal of nonhuman primates. J Lipid Res 2000;41:1991-2001.

22. Temel RE, Gebre AK, Parks JS, Rudel LL. Compared with Acyl-CoA:cholesterol O-acyltransferase (ACAT) 1 and lecithin:cholesterol acyltransferase, ACAT2 displays the greatest capacity to differentiate cholesterol from sitosterol. J Biol Chem 2003;278:47594-601.

23. Salen G, Horak I, Rothkopf M, Cohen JL, Speck J, Tint GS, et al. Lethal atherosclerosis associated with abnormal plasma and tissue sterol composition in sitosterolemia with xanthomatosis. J Lipid Res 1985;26:1126-33.

24. Miettinen TA, Klett EL, Gylling H, Isoniemi H, Patel SB. Liver transplantation in a patient with sitosterolemia and cirrhosis. Gastroenterology 2006;130:542-7.

25. Wang J, Mitsche MA, Lutjohann D, Cohen JC, Xie XS, Hobbs HH. Relative roles of ABCG5/ABCG8 in liver and intestine. J Lipid Res 2015;56:319-30.

26. Myrie SB, Mymin D, Triggs-Raine B, Jones PJ. Serum lipids, plant sterols, and cholesterol kinetic responses to plant sterol supplementation in phytosterolemia heterozygotes and control individuals. Am J Clin Nutr 2012;95:837-44.

27. Bhattacharyya AK, Connor WE. Beta-sitosterolemia and xanthomatosis. A newly described lipid storage disease in two sisters. J Clin Invest 1974;53:1033-43.

28. Wang J, Joy T, Mymin D, Frohlich J, Hegele RA. Phenotypic heterogeneity of sitosterolemia. J Lipid Res 2004;45:2361-7.

29. Hu M, Yuen YP, Kwok JS, Griffith JF, Tomlinson B. Potential effects of NPC1L1 polymorphisms in protecting against clinical disease in a chinese family with sitosterolaemia. J Atheroscler Thromb 2014;21:989-95.

30. Hansel B, Carrié A, Brun-Druc N, Leclert G, Chantepie $S$, Coiffard AS, et al. Premature atherosclerosis is not systematic in phytosterolemic patients: severe hypercholesterolemia as a confounding factor in five subjects. Atherosclerosis 2014;234:162-8.

31. Mannucci L, Guardamagna O, Bertucci P, Pisciotta L, 
Liberatoscioli L, Bertolini S, et al. Beta-sitosterolaemia: a new nonsense mutation in the ABCG5 gene. Eur J Clin Invest 2007;37:997-1000.

32. Niu DM, Chong KW, Hsu JH, Wu TJ, Yu HC, Huang CH, et al. Clinical observations, molecular genetic analysis, and treatment of sitosterolemia in infants and children. J Inherit Metab Dis 2010;33:437-43.

33. Rios J, Stein E, Shendure J, Hobbs HH, Cohen JC. Identification by whole-genome resequencing of gene defect responsible for severe hypercholesterolemia. Hum Mol Genet 2010;19:4313-8.

34. Drozdowski LA, Clandinin T, Thomson AB. Ontogeny, growth and development of the small intestine: Understanding pediatric gastroenterology. World J Gastroenterol 2010;16:787-99.

35. Park JH, Chung IH, Kim DH, Choi MH, Garg A, Yoo EG. Sitosterolemia presenting with severe hypercholesterolemia and intertriginous xanthomas in a breastfed infant: case report and brief review. J Clin Endocrinol Metab 2014;99:1512-8.

36. Kamelska AM, Pietrzak-Fiecko R, Bryl K. Variation of the cholesterol content in breast milk during 10 days collection at early stages of lactation. Acta Biochim Pol 2012;59:243-7.

37. Sethuraman G, Sugandhan S, Sharma G, Chandramohan K, Chandra NC, Dash SS, et al. Familial homozygous hypercholesterolemia: report of two patients and review of the literature. Pediatr Dermatol 2007;24:230-4.

38. Canizales-Quinteros S, Aguilar-Salinas CA, HuertasVazquez A, Ordonez-Sanchez ML, Rodriguez-Torres M, Venturas-Gallegos JL, et al. A novel ARH splice site mutation in a Mexican kindred with autosomal recessive hypercholesterolemia. Hum Genet 2005;116:114-20.

39. Manchanda Y, Sharma VK. Intertriginous xanthomas: a marker of homozygous type Ila hyperlipoproteinemia. Int J Dermatol 2004;43:676-7.

40. Zak A, Zeman M, Slaby A, Vecka M. Xanthomas: clinical and pathophysiological relations. Biomed Pap Med Fac Univ Palacky Olomouc Czech Repub 2014;158:181-8.

41. Oosterveer DM, Versmissen J, Yazdanpanah M, Hamza $\mathrm{TH}$, Sijbrands EJ. Differences in characteristics and risk of cardiovascular disease in familial hypercholesterolemia patients with and without tendon xanthomas: a systematic review and meta-analysis. Atherosclerosis 2009;207:311-7.

42. Menotti A, Puddu PE, Lanti M, Maiani G, Fidanza F. Cardiovascular risk factors predict survival in middle-aged men during 50 years. Eur J Intern Med 2013;24:67-74.

43. Belamarich PF, Deckelbaum RJ, Starc TJ, Dobrin BE, Tint GS, Salen G. Response to diet and cholestyramine in a patient with sitosterolemia. Pediatrics 1990;86:977-81.

44. Parsons HG, Jamal R, Baylis B, Dias VC, Roncari D. A marked and sustained reduction in LDL sterols by diet and cholestyramine in beta-sitosterolemia. Clin Invest Med 1995; 18:389-400.

45. Salen G, Starc T, Sisk CM, Patel SB. Intestinal cholesterol absorption inhibitor ezetimibe added to cholestyramine for sitosterolemia and xanthomatosis. Gastroenterology 2006;130:1853-7.

46. Cheng WF, Yuen YP, Chow CB, Au KM, Chan YW, Tam SC. Sitosterolaemia and xanthomatosis in a child. Hong Kong Med J 2003;9:206-9.

47. Mymin D, Wang J, Frohlich J, Hegele RA. Image in cardiovascular medicine. Aortic xanthomatosis with coronary ostial occlusion in a child homozygous for a nonsense mutation in ABCG8. Circulation 2003;107:791.

48. Kwiterovich PO Jr, Bachorik PS, Smith HH, McKusick VA, Connor WE, Teng B, et al. Hyperapobetalipoproteinaemia in two families with xanthomas and phytosterolaemia. Lancet 1981;1:466-9.

49. Falk E, Shah PK, Fuster V. Coronary plaque disruption. Circulation 1995;92:657-71.

50. Bao L, Li Y, Deng SX, Landry D, Tabas I. Sitosterolcontaining lipoproteins trigger free sterol-induced caspaseindependent death in ACAT-competent macrophages. J Biol Chem 2006;281:33635-49.

51. Kolovou G, Voudris V, Drogari E, Palatianos G, Cokkinos DV. Coronary bypass grafts in a young girl with sitosterolemia. Eur Heart J 1996;17:965-6.

52. Su Y, Wang Z, Yang H, Cao L, Liu F, Bai X, et al. Clinical and molecular genetic analysis of a family with sitosterolemia and co-existing erythrocyte and platelet abnormalities. Haematologica 2006;91:1392-5.

53. Wang Z, Cao L, Su Y, Wang G, Wang R, Yu Z, et al. Specific macrothrombocytopenia/hemolytic anemia associated with sitosterolemia. Am J Hematol 2014;89:320-4.

54. Kaya Z, Niu DM, Yorulmaz A, Tekin A, Gursel T. A novel mutation of ABCG5 gene in a Turkish boy with phytosterolemia presenting with macrotrombocytopenia and stomatocytosis. Pediatr Blood Cancer 2014;61:1457-9.

55. Kanaji T, Kanaji S, Montgomery RR, Patel SB, Newman PJ. Platelet hyperreactivity explains the bleeding abnormality and macrothrombocytopenia in a murine model of sitosterolemia. Blood 2013;122:2732-42.

56. Honda A, Salen G, Nguyen LB, Tint GS, Batta AK, Shefer S. Down-regulation of cholesterol biosynthesis in sitosterolemia: diminished activities of acetoacetyl-CoA thiolase, 3-hydroxy-3-methylglutaryl-CoA synthase, reductase, squalene synthase, and 7-dehydrocholesterol delta7reductase in liver and mononuclear leukocytes. J Lipid Res 1998;39:44-50.

57. Plat J, Nichols JA, Mensink RP. Plant sterols and stanols: effects on mixed micellar composition and LXR (target gene) activation. J Lipid Res 2005;46:2468-76.

58. Yang C, Yu L, Li W, Xu F, Cohen JC, Hobbs HH. Disruption of cholesterol homeostasis by plant sterols. J Clin Invest 2004;114:813-22.

59. Nguyen L, Salen G, Shefer S, Shore V, Tint GS, Ness G. Unexpected failure of bile acid malabsorption to stimulate cholesterol synthesis in sitosterolemia with xanthomatosis. Comparison with lovastatin. Arteriosclerosis 1990;10:28997. 
60. Expert Panel on Integrated Guidelines for Cardiovascular Health and Risk Reduction in Children and Adolescents; National Heart, Lung, and Blood Institute. Expert panel on integrated guidelines for cardiovascular health and risk reduction in children and adolescents: summary report. Pediatrics 2011;128 Suppl 5:S213-56.

61. Lutjohann D, von Bergmann K, Sirah W, Macdonell G, Johnson-Levonas AO, Shah A, et al. Long-term efficacy and safety of ezetimibe $10 \mathrm{mg}$ in patients with homozygous sitosterolemia: a 2-year, open-label extension study. Int J Clin Pract 2008;62:1499-510.

62. Gregg RE, Connor WE, Lin DS, Brewer HB Jr. Abnormal metabolism of shellfish sterols in a patient with sitosterolemia and xanthomatosis. J Clin Invest 1986;77: 1864-72.

63. Tsubakio-Yamamoto K, Nishida M, Nakagawa-Toyama Y, Masuda D, Ohama T, Yamashita S. Current therapy for patients with sitosterolemia: effect of ezetimibe on plant sterol metabolism. J Atheroscler Thromb 2010;17:891-900.
64. Salen G, von Bergmann K, Lutjohann D, Kwiterovich P, Kane J, Patel SB, et al. Ezetimibe effectively reduces plasma plant sterols in patients with sitosterolemia. Circulation 2004;109:966-71.

65. Othman RA, Myrie SB, Mymin D, Merkens LS, Roullet JB, Steiner RD, et al. Ezetimibe reduces plant sterol accumulation and favorably increases platelet count in sitosterolemia. J Pediatr 2015;166:125-31.

66. Kavey RE, Allada V, Daniels SR, Hayman LL, McCrindle BW, Newburger JW, et al. Cardiovascular risk reduction in high-risk pediatric patients: a scientific statement from the American Heart Association Expert Panel on Population and Prevention Science; the Councils on Cardiovascular Disease in the Young, Epidemiology and Prevention, Nutrition, Physical Activity and Metabolism, High Blood Pressure Research, Cardiovascular Nursing, and the Kidney in Heart Disease; and the Interdisciplinary Working Group on Quality of Care and Outcomes Research: endorsed by the American Academy of Pediatrics. Circulation 2006;114:2710-38. 